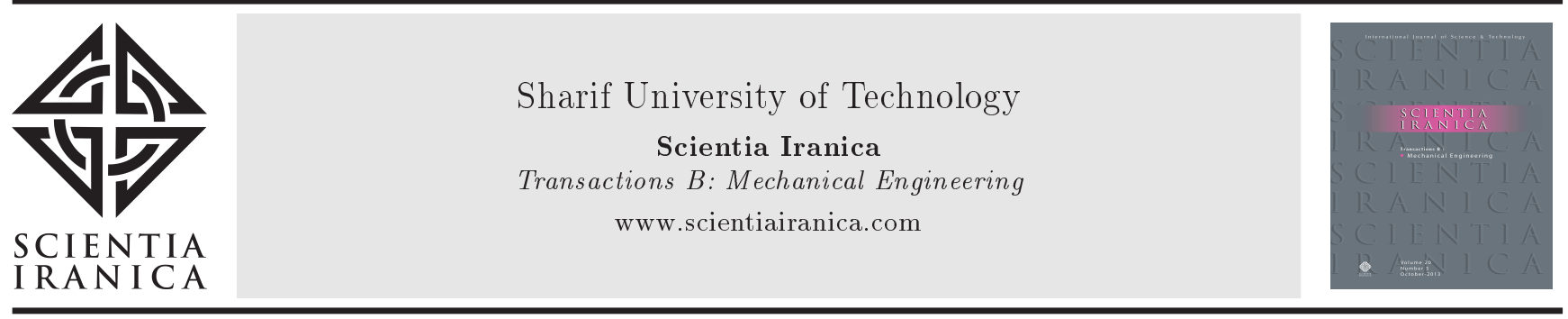

Research Note

\title{
Magnetohydrodynamic flow of linear visco-elastic fluid model above a shrinking/stretching sheet: A series solution
}

\author{
Y. Khan* \\ Department of Mathematics, Zhejiang University, Hangzhou 310027, China. \\ Received 17 June 2014; received in revised form 8 June 2016; accepted 13 August 2016
}

\author{
KEYWORDS \\ Linear visco-elastic \\ fluid; \\ Boundary layer \\ approximations; \\ Homotopy \\ Perturbation Method \\ (HPM); \\ Finite difference \\ technique; \\ Magneto- \\ hydrodynamics \\ (MHD); \\ Shrinking/stretching \\ sheet.
}

\begin{abstract}
In this paper, a series solution is obtained for MHD flow of linear viscoelastic fluid over a shrinking/stretching sheet by using Homotopy Perturbation Method (HPM). The governing Navier-Stokes equations of the flow are transformed to an ordinary differential equation by a suitable similarity transformation and stream function. The influence of various parameters such as Hartman number and Deborah number on the velocity field is analyzed by appropriate graphs. Finally, the validity of results is verified by comparing them with numerical results. Results are presented graphically and in tabulated forms to study the efficiency and accuracy of the homotopy perturbation method.
\end{abstract}

(C) 2017 Sharif University of Technology. All rights reserved.

\section{Introduction}

A fundamental visco-elastic model of differential type is the Upper Convected Maxwell fluid (UCM), which models the polymer contribution of some types of Boger fluids [1] and polymer melts of constant viscosity. The visco-elastic fluid models are already used in simple models such as second-order model and/or Walters' B model, which are known to be good only for weakly elastic fluids [1] subject to slow and/or slowly varying flows. To this should be added the fact that these two fluid models [2] are known to violate certain rules of thermodynamics. Another shortcoming of the above models is in the notion that virtually

\footnotetext{
*.E-mail address: yasirmath@yahoo.com
}

all of them are based on the use of boundary layer theory, which is still incomplete for non-Newtonian fluids [3]. Therefore, the significance of the results reported in the previous studies is limited, at least as far as polymer industry is concerned. Obviously, for the theoretical results to become of any industrial significance, more realistic visco-elastic fluid models such as upper-convected Maxwell model or Oldroyd-B model should be invoked in the analysis. The Maxwell model [4] is capable of describing stress relaxation effects and has been applied to problems having small dimensionless relation time. However, the model does not fully account for the elasticity of the fluid and typically fails to predict retardation effects as it lacks the retardation timescale that characterizes other visco-elastic models such as Jeffrey's model [4-7]. The Linear visco-elastic fluids such as Upper-Convected Maxwell fluid (UCM) have been extensively utilized 
in various aspects, for example in MHD flows, Porous plate, porous medium, stretching and non-stretching sheets, and heat transfer effects. The literature on the topic is quite extensive and, hence, cannot be described here in detail. However, some most recent works of eminent researchers regarding the UCM fluid may be mentioned [8-19].

In this study, we have applied He's HPM to find the series solution to nonlinear differential equation governing visco-elastic fluid boundary-layer problems. The Homotopy Perturbation Method (HPM) was proposed by He [20-22] in 1999. In this method, the solution is considered as the summation of an infinite series, which usually converge rapidly on the exact solution. Using the homotopy technique from topology, a homotopy is constructed with an embedding parameter, $p \in[0,1]$, which is considered as a "small parameter". The approximations obtained by the homotopy perturbation method are uniformly valid not only for small parameters, but also for very large parameters. The HPM continuously deforms a difficult problem into a simple one, which is easy to solve. It has been shown by many authors that this method provides improvements to the existing numerical techniques. Considerable research [23-30] has been recently conducted on applying this method to a wide class of linear and nonlinear equations. However, to the best of the author's knowledge, no one has studied the MHD flow of a linear visco-elastic fluid above a shrinking/stretching sheet by means of homotopy perturbation method.

\section{Formulation of the problem}

Consider the MHD flow of an incompressible linear visco-elastic fluid above a shrinking/stretching sheet. The flow in the fluid system occurs due to shrinking/stretching of a plate. It is governed by the following equations:

$$
\begin{aligned}
& \operatorname{div}=0 \\
& \begin{aligned}
u \frac{\partial u}{\partial x} & +\nu \frac{\partial u}{\partial y}+\lambda\left[u^{2} \frac{\partial^{2} u}{\partial x^{2}}+\nu^{2} \frac{\partial^{2} u}{\partial y^{2}}+2 u \nu \frac{\partial^{2} u}{\partial x \partial y}\right] \\
& =\gamma \frac{\partial^{2} u}{\partial y^{2}}-\frac{\sigma B_{0}^{2}}{\rho} u .
\end{aligned}
\end{aligned}
$$

The appropriate boundary conditions are:

$$
\begin{aligned}
& u=U, \quad \nu=0 \quad \text { at } \quad y=0, \\
& u \rightarrow 0 \quad \text { at } \quad y \rightarrow \infty .
\end{aligned}
$$

Taking into account the stream function and similarity transformation:

$$
\begin{aligned}
u & =\frac{\partial \psi}{\partial y}, \quad \nu=-\frac{\partial \psi}{\partial x}, \quad \psi=\sqrt{\gamma x U} f(\eta), \\
\eta & =\sqrt{\frac{U}{\gamma x} y,}
\end{aligned}
$$

Eq. (1) is automatically satisfied and Eqs. (2) and (3) are reduced to:

$$
\begin{aligned}
& 2 f^{\prime \prime \prime}-2 M^{2} f^{\prime}+f f^{\prime \prime} \\
& \quad-\beta\left(2 f f^{\prime} f^{\prime \prime}+f^{2} f^{\prime \prime \prime}+\eta\left(f^{\prime}\right)^{2} f^{\prime \prime}\right)=0 .
\end{aligned}
$$

Corresponding boundary condition takes the form:

$$
\begin{array}{ll}
f(0)=0, & f^{\prime}(0)=-1, \\
f^{\prime} \rightarrow 0, & \eta \rightarrow \infty
\end{array}
$$

where:

$$
\beta=\frac{\lambda U}{2 x}, \quad M^{2}=\frac{\sigma B_{0}^{2} x}{\rho U} .
$$

In view of HPM [20-22], Eq. (5) is expressed as:

$$
\begin{aligned}
& (1-p) L\left(f-f_{0}\right)+p\left(f^{\prime \prime \prime}-M^{2} f^{\prime}+\frac{1}{2} f f^{\prime \prime}\right. \\
& \left.-\frac{\beta}{2}\left(2 f f^{\prime} f^{\prime \prime}+f^{2} f^{\prime \prime \prime}+\eta\left(f^{\prime}\right)^{2} f^{\prime \prime}\right)\right)=0, \\
& f=f_{0}+p f_{1}+p^{2} f_{2}+\cdots
\end{aligned}
$$

Assuming $L f=0$, substituting $f$ from Eq. (9) into Eq. (8), and making some simplification and rearrangement based on powers of $p$-terms, we have:

$$
\begin{aligned}
& p^{(0)}: \quad L f_{0}=0 \\
& f_{0}(0)=0, \quad f_{0}^{\prime}(0)=-1, \quad f_{0}^{\prime}(\infty)=0,
\end{aligned}
$$

where $L$ is defined as:

$$
L=\frac{\partial^{3}}{\partial \eta^{3}}+\frac{\partial^{2}}{\partial \eta^{2}} \text {. }
$$

On solving Eq. (10), we get the initial guess as follows:

$$
\begin{aligned}
& f_{0}(\eta)=1+e^{-\eta} \\
& \begin{array}{l}
p^{(1)}: \quad L f_{j}+f_{j-1}^{\prime \prime \prime}-M^{2} f_{0}^{\prime}+\frac{1}{2} f_{0} f_{0}^{\prime \prime} \\
\quad-\frac{\beta}{2}\left(2 f_{0} f_{0}^{\prime} f_{0}^{\prime \prime}+f_{0}^{2} f_{0}^{\prime \prime \prime}+\eta\left(f_{0}^{\prime}\right)^{2} f_{0}^{\prime \prime}\right)=0 \\
f_{1}(0)=0, \quad f_{1}^{\prime}(0)=0, \quad f_{1}^{\prime}(\infty)=0
\end{array}
\end{aligned}
$$




$$
\begin{aligned}
& p^{(j)}: \quad L f_{j}+f_{j-1}^{\prime \prime \prime}-M^{2} f_{j-1}^{\prime}+\frac{1}{2} \sum_{k=0}^{j-1} f_{k}^{\prime} f_{j-1-k}^{\prime \prime} \\
&-\frac{\beta}{2}\left(2 \sum_{k=0}^{m-1} f_{m-1-k} \sum_{l=0}^{k} f_{k-l}^{\prime} f_{l}^{\prime \prime}\right. \\
&+\sum_{k=0}^{m-1} f_{m-1-k} \sum_{l=0}^{k} f_{k-l} f_{l}^{\prime \prime \prime} \\
&\left.+\eta \sum_{k=0}^{m-1} f_{m-1-k}^{\prime} \sum_{l=0}^{k} f_{k-l}^{\prime} f_{l}^{\prime \prime}\right)=0 \\
& f_{j}(0)=0, \quad f_{j}^{\prime}(0)=0, \quad f_{j}^{\prime}(\infty)=0 .
\end{aligned}
$$

On solving Eqs. (13) and (14) in any software like MATHEMATICA, MAPLE, or MATLAB, we write the first-order approximation:

$$
\begin{aligned}
f= & \frac{689}{576}+\frac{5 e^{-4 \eta}}{576}+\frac{e^{-3 \eta}}{36}+\frac{e^{-2 \eta}}{8}-\frac{391 e^{-\eta}}{288} \\
& -\frac{M^{2}}{4}-\frac{M^{2} e^{-2 \eta}}{4}+\frac{M^{2} e^{-\eta}}{2}+\frac{\beta}{288}-\frac{\beta e^{-4 \eta}}{32} \\
& +\frac{\beta e^{-3 \eta}}{9}-\frac{\beta e^{-2 \eta}}{8}+\frac{\beta e^{-\eta}}{24}+\frac{\eta e^{-4 \eta}}{96} .
\end{aligned}
$$

\subsection{Stretching sheet problem}

For stretching phenomena, the governing equation is (5); however, the boundary conditions for the stretching case are as follows:

$$
\begin{array}{ll}
f(0)=0, & f^{\prime}(0)=1, \\
f^{\prime} \rightarrow 0, & \eta \rightarrow \infty .
\end{array}
$$

The solution to the above boundary value problem has been calculated using the procedure discussed in the previous section on shrinking. To avoid the repetition, the complete solution is not defined; however, the initial guesses are as follows:

$$
f_{0}(\eta)=1-e^{-\eta}
$$

\section{Results and discussion}

MHD flow of linear visco-elastic fluid over a shrinking/stretching sheet is analytically studied. Validity of the homotopy perturbation method is shown in Table 1 and Figures 1 and 2 for shrinking/stretching sheet. Computations are carried out for a wide range of physical parameters of the problem and the graphical results are presented to illustrate the effects of various
Table 1. Comparison between HPM and finite difference results of $f(\eta)$.

\begin{tabular}{cccc}
\hline $\boldsymbol{\eta}$ & Present method & Numerical & Error \\
\hline 0 & 0 & 0 & 0 \\
1 & 0.6061 & 0.6052 & 0.0008 \\
2 & 0.7856 & 0.7867 & 0.0011 \\
3 & 0.8269 & 0.8343 & 0.0073 \\
4 & 0.8319 & 0.8431 & 0.0112 \\
\hline
\end{tabular}

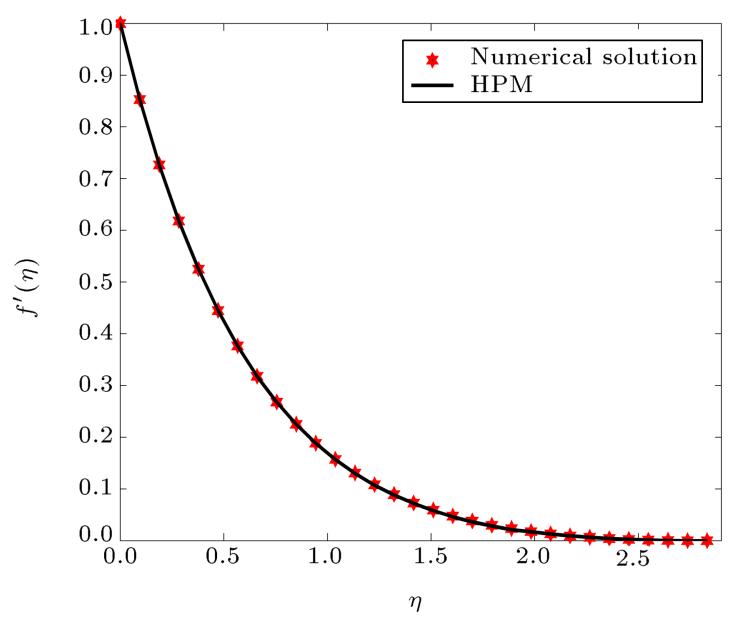

Figure 1. Graphical comparison between HPM and finite difference solutions for the stretching case.

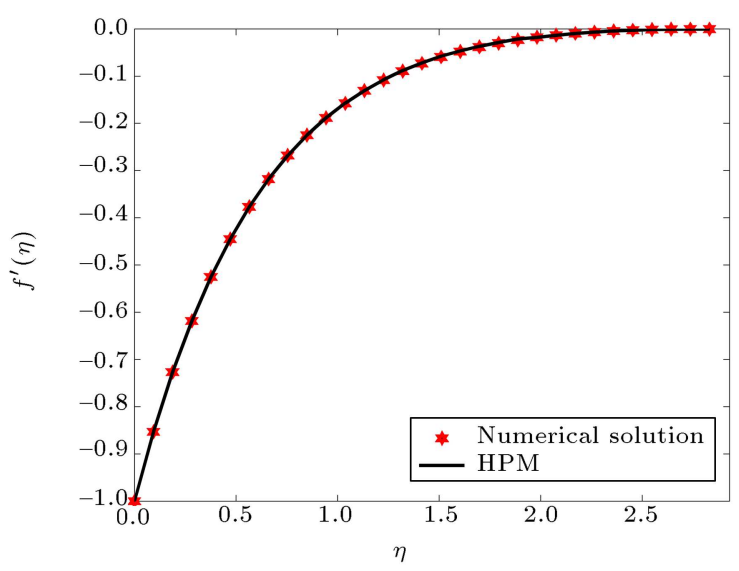

Figure 2. Graphical comparison between HPM and finite difference solutions for the shrinking case.

controlling parameters, including Hartman number $(M)$ and Deborah number $(\beta)$. Figures 3 to 6 are provided to show the effects of the Deborah number, $\beta$, on the velocity components $f^{\prime}$ and $f$ for shrinking and stretching, respectively. It is found from Figure 3 that $f^{\prime}$ decreases by increasing $\beta$ for shrinking case. Figure 4 shows the behavior of $\beta$ on $f^{\prime}$; it is found that boundary layer thickness decreases with an increase in $\beta$ for stretching case. To show the influence of $\beta$ on the velocity component, $f$, Figures 5 and 6 are plotted for shrinking and stretching, respectively. It 


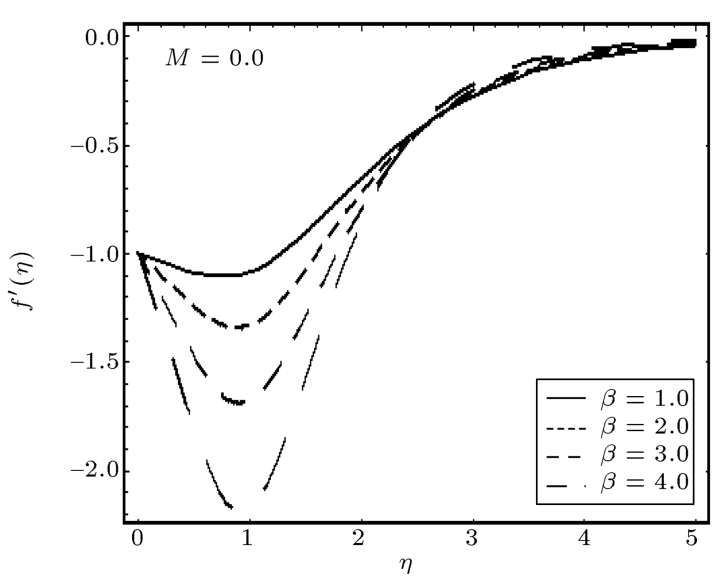

Figure 3. Variation of $f^{\prime}$ for different values of $\beta$ (shrinking case).

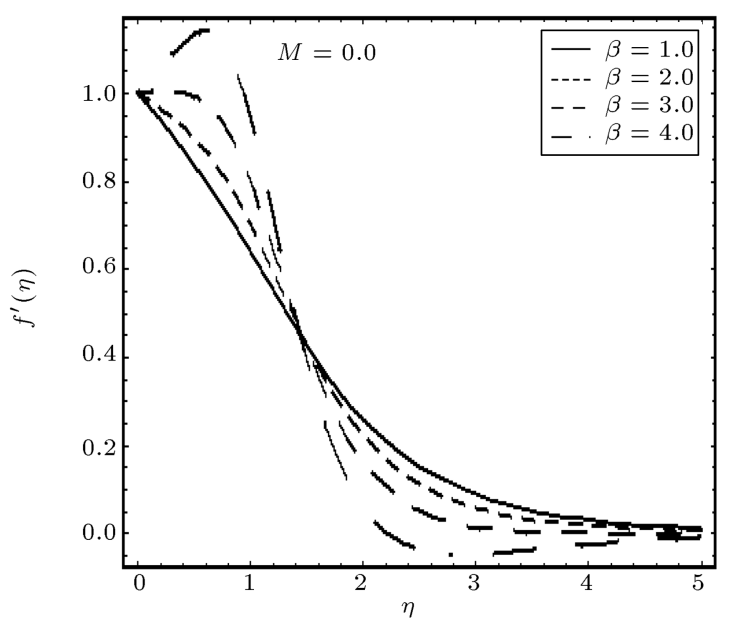

Figure 4. Variation of $f^{\prime}$ for different values of $\beta$ (stretching case).

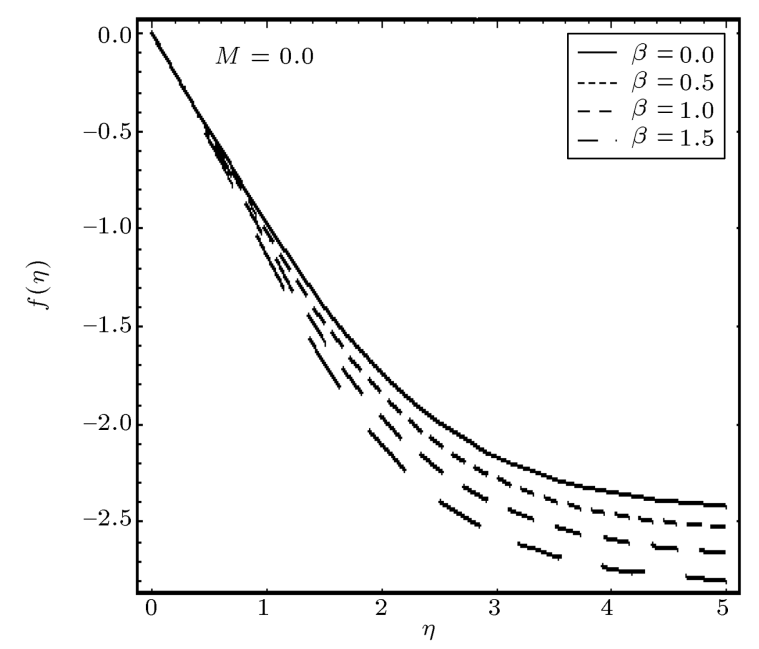

Figure 5. Variation of $f$ for different values of $\beta$ (shrinking case).

is depicted in Figures 5 and 6 that behavior of $f$ is quite similar to that of $f^{\prime}$ for the shrinking/stretching case. Figures 7 to 10 show the behavior of the velocity

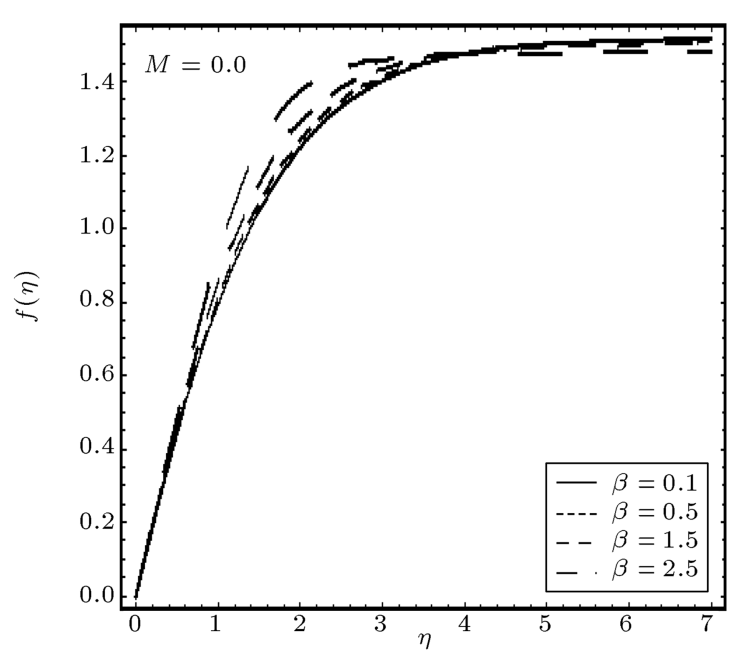

Figure 6. Variation of $f$ for different values of $\beta$ (stretching case).

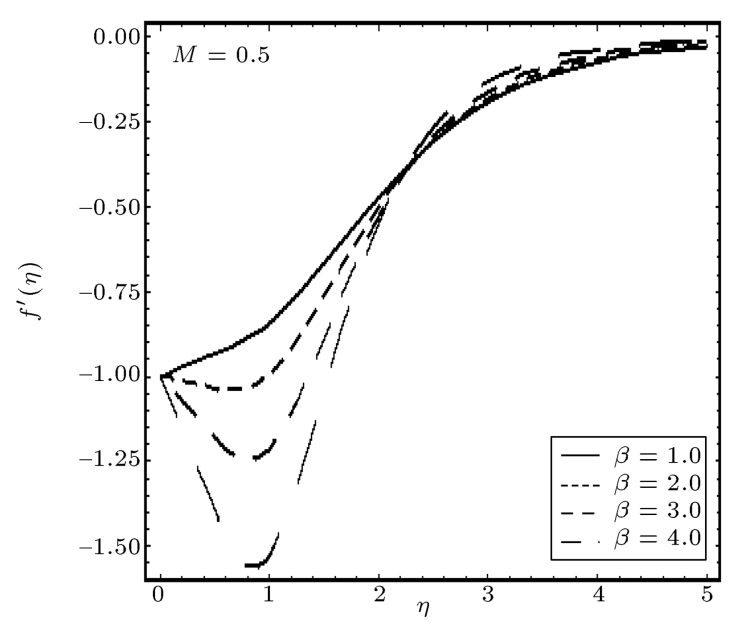

Figure 7. Variation of $f^{\prime}$ for different values of $\beta$ (shrinking case).

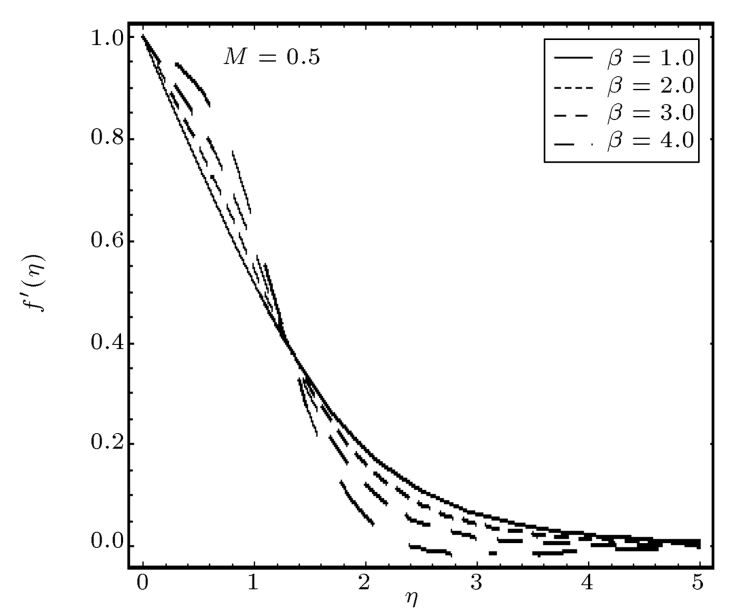

Figure 8. Variation of $f^{\prime}$ for different values of $\beta$ (stretching case).

components when the flow is considered in the presence of a uniformly imposed magnetic field. It is evident that the introduction of the magnetic field strengthens 


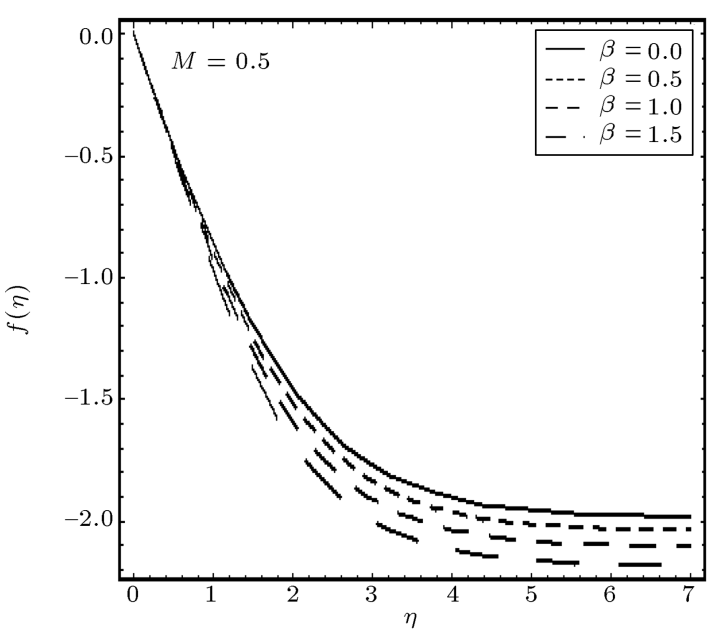

Figure 9. Variation of $f$ for different values of $\beta$ (shrinking case).

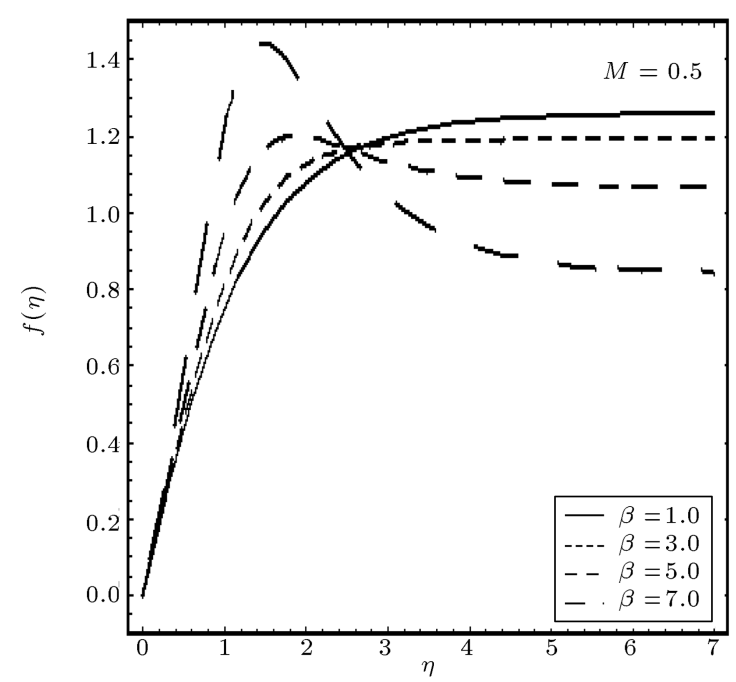

Figure 10. Variation of $f$ for different values of $\beta$ (stretching case).

the thinning effect. The effects of MHD parameter are presented in Figures 11 to 14 for both shrinking and stretching. It is observed that $f^{\prime}$ increases with the increase in $M$ in the shrinking case (see Figure 11); however, velocity decreases and the boundary-layer thickness increases for the $x$-component of velocity in the stretching case (see Figure 12). In the shrinking case, velocity component, $f$, shows quite opposite behavior to that of $f^{\prime}$ for $M$ in Figure 13, whereas the $y$-component of the velocity decreases and boundarylayer thickness increases for large values of the MHD parameter.

\section{Conclusion}

The MHD flow of an incompressible visco-elastic fluid due to shrinking/stretching surface is examined. The governing equations are analytically solved using the

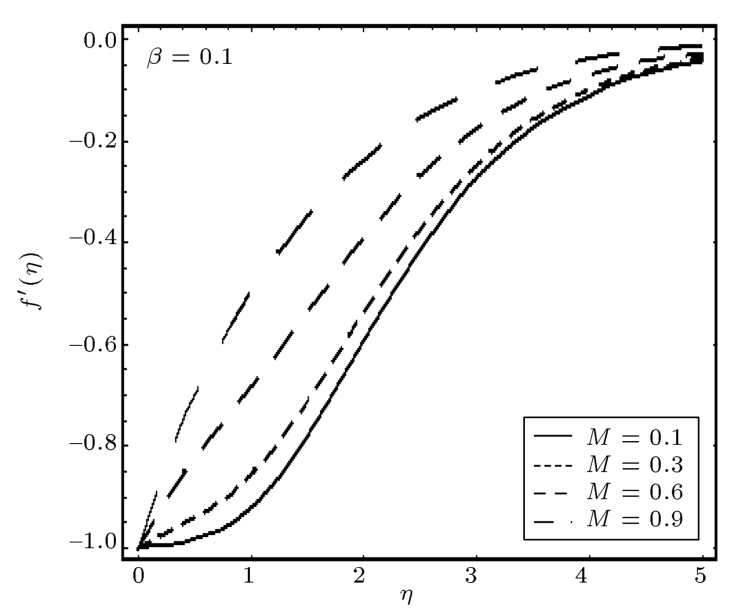

Figure 11. Variation of $f^{\prime}$ for different values of $M$ (shrinking case).

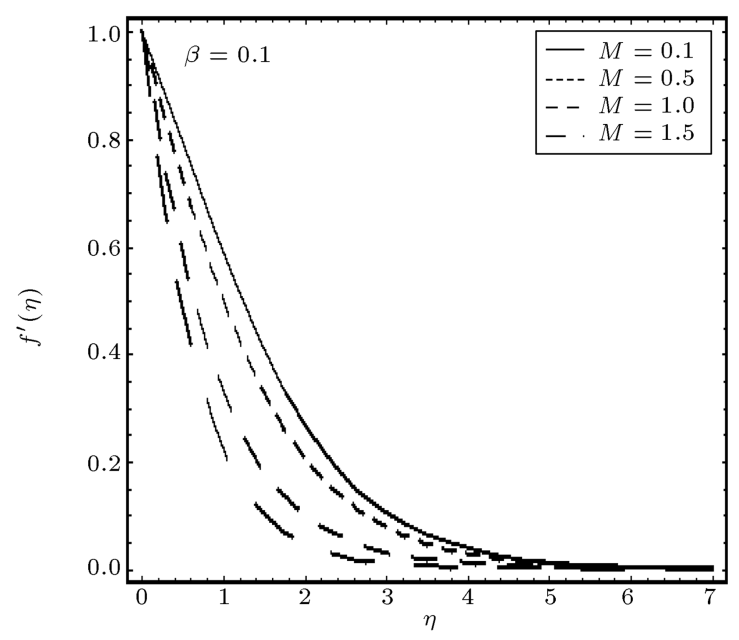

Figure 12. Variation of $f^{\prime}$ for different values of $M$ (stretching case).

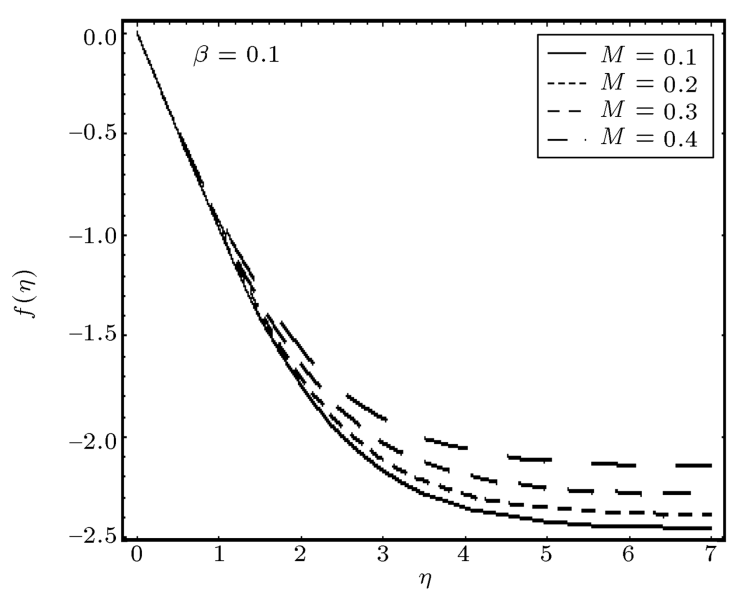

Figure 13. Variation of $f$ for different values of $M$ (shrinking case).

homotopy perturbation method for the first time. The results have been presented in the series form. The influence of constant parameters such as Deborah num- 


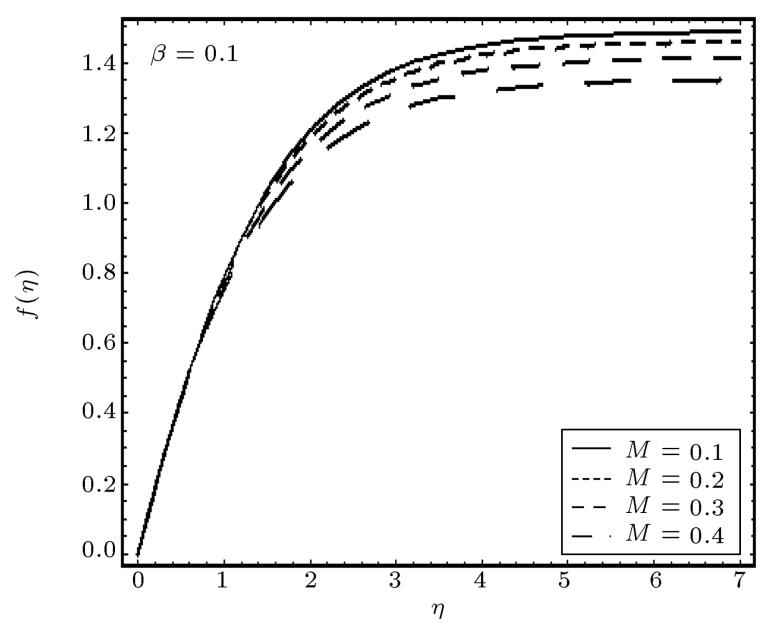

Figure 14. Variation of $f$ for different values of $M$ (stretching case).

ber and MHD on the velocity field is discussed. Also, the property of the paper is the error analysis between the approximate solutions and numerical solutions, which shows that our approximate solutions converge very rapidly on the numerical solutions.

\section{Nomenclature}

$\rho \quad$ Density of fluid

$\gamma \quad$ Kinematic viscosity

$U \quad$ Reference velocity

$f \quad$ Dimensionless velocity profile

$u \quad$ Velocity component in $x$ direction

$\nu \quad$ Velocity component in $y$ direction

$\eta \quad$ Independent dimensionless parameter

$B_{0} \quad$ Uniform static magnetic field

$\sigma \quad$ Electrical conductivity

$\beta \quad$ Deborah number

$M \quad$ Hartman number

$\lambda \quad$ Material parameter (ratio of relaxation to retardation times)

\section{References}

1. Bird, R.B., Armstrong, R.A. and Hassager, O., Dynamics of Polymeric Liquids, I: Fluid Dynamics, John Wiley and Sons (1987).

2. Fosdick, R.L. and Rajagopal, K.R. "Anomalous features in the model of second-order fluids", Arch. Ration. Mech. Anal., 70, pp. 145-152 (1979).

3. Gupta, A.S. and Wineman, A.S. "On a boundary layer theory for non-Newtonian fluids", Lett. Appl. Eng. Sci., 18, pp. 875-883 (1980).

4. Mardones, J.M. and Garcia, C.P. "Linear instability in viscoelastic fluid convection", J. Phys.: Condens. Matter, 2, pp. 1281-1290 (1990).
5. Maxwell, J.C. "On the dynamical theory of gases", Philos. Trans. R. Soc. Lond. A, 157, pp. 49-88 (1867).

6. Eltayeb, I.A. "Nonlinear thermal convection in an elasticoviscous layer heated from below", Proc. R. Soc. Lond. A, 356, pp. 161-176 (1977).

7. Sokolov, M. and Tanner, R.I. "Convective stability of a general viscoelastic fluid heated from below", Phys. Fluid, 15, pp. 534-539 (1972).

8. Wang, S.W. and Tan, W.C. "Stability analysis of double-diffusive convection of Maxwell fluid in a porous medium heated from below", Phys. Lett. A, 372, pp. 3046-3050 (2008).

9. Wang, S.W. and Tan, W.C. "The onset of DarcyBrinkman thermosolutal convection in a horizontal porous media", Phys. Lett. A, 373, pp. 776-780 (2009).

10. Sadeghy, K., Najafi, A.H. and Saffaripour, M. "Sakiadis flow of an upper-convected Maxwell fluid", Int. J. Non-Linear Mech., 40, pp. 1220-1228 (2005).

11. Sadeghy, K., Khabazi, N. and Taghavi, S.M. "Magnetohydrodynamic (MHD) flows of viscoelastic fluids in converging/diverging channels", Int. J. Eng. Sci., 45, pp. 923-938 (2007).

12. Pahlavan, A.A., Aliakbar, V., Farahani, F.V. and Sadeghy, K. "MHD flows of UCM fluids above porous stretching sheets using two-auxiliary-parameter homotopy analysis method", Commun Nonlinear Sci. Numer. Simulat., 14, pp. 473-488 (2009).

13. Aliakbar, V., Pahlavan, A.A. and Sadeghy, K. "The influence of thermal radiation on MHD flow of Maxwellian fluids above stretching sheets", Communications in Nonlinear Science and Numerical Simulation, 14, pp. 779-794 (2009).

14. Pahlavan, A.A. and Sadeghy, K. "On the use of homotopy analysis method for solving unsteady MHD flow of Maxwellian fluids above impulsively stretching sheets", Commun Nonlinear Sci. Numer. Simulat., 14, pp. 1355-1365 (2009).

15. Raftari, B. and Yildirim, A. "The application of homotopy perturbation method for MHD flows of UCM fluids above porous stretching sheets", Computers and Mathematics with Applications, 59, pp. 3328-3337 (2010).

16. Sekhar, G.N. and Jayalatha, G. "Elastic effects on Rayleigh-Bérnard convection in liquids with temperature-dependent viscosity", Int. J. Thermal Sci., pp. 67-75 (2010).

17. Agadi, A.H., Abel, M.S., Tawade, J.V. and Maharudrappa, I. "Effect of non-uniform heat source for the UCM fluid over a stretching sheet with magnetic field", Int. J. Adv. Resear. Eng. Tech., 4, pp. 40-49 (2013).

18. Abel, M.S., Tawade, J.V. and Shinde, J.N. "The effects of MHD flow and heat transfer for the UCM fluid over a stretching surface in presence of thermal radiation", Adv. Math. Phys., 2012, p. 702681, 21 pages (2012).

19. Babaelahi, M., Ganji, D.D. and Joneidi, A.A. "Viscoelastic MHD flow boundary layer over a stretching 
surface with viscous and ohmic dissipations", Meccanca, 45, pp. 817-827 (2010)

20. He, J.H. "Homotopy perturbation technique", Сomp. Meth. Appl. Mech. Engrg., 178, pp. 257-262 (1999).

21. He, J.H. "A coupling method of a homotopy technique and a perturbation technique for non-linear problems", Internat. J. Non-linear Mech., 35, pp. 37-43 (2000).

22. He, J.H. "Homotopy perturbation method: A new nonlinear analytical technique", Appl. Math. Comput., 135, pp. 73-79 (2003).

23. Eslami, M., Soltanalizadeh, B. and Boubaker, K., Enhanced Homotopy Perturbation Method (EHPM) and Boubaker Polynomials Expansion Scheme (BPES) Comparative Solutions to Partial Differential Equations Systems Governing Non-isothermal Tubular Chemical Reactors, 3, pp. 219-226 (2013).

24. Hetmaniok, E., Nowak, I., Slota, D., Wituła, R. "Application of the homotopy perturbation method for the solution of inverse heat conduction problem", International Communications in Heat and Mass Transfer, 39, pp. 30-35 (2012).

25. Khan, Y., Wu, Q., Faraz, N. and Yildirim, A. "The effects of variable viscosity and thermal conductivity on a thin film flow over a shrinking/stretching sheet", Computers and Mathematics with Applications, 61, pp. 3391-3399 (2011).

26. Khan, Y. and Wu, Q. "Homotopy Perturbation transform method for nonlinear equations using He's polynomials", Computers and Mathematics with Applications, 61, pp. 1963-1967 (2011).
27. Khan, Y., Faraz, N., Yildirim, A. and Wu, Q. "A series solution of the long porous slider", Tribology Transactions, 54, pp. 187-191 (2011).

28. Turkyilmazoglu, M. "Analytic approximate solutions of parameterized unperturbed and singularly perturbed boundary value problems", Applied Mathematical Modelling, 35, pp. 3879-3886 (2011).

29. Ziabakhsh, Z., Domairry, G., Domiri, A. and Moghimi, S.M. "New applications of approximate methods in fluid mechanics", Asia-Pacific Journal of Chemical Engineering, 6, pp. 669-678 (2011).

30. Ghorbani, A. and Nadjafi, J.S. "Exact solutions for nonlinear integral equations by a modified homotopy perturbation method", Comput. Math. Appl., 56, pp. 1032-1039 (2008).

\section{Biography}

Yasir Khan obtained his PhD from Zhejiang University, China. He is the Editor of three international journals. His current research mainly covers analytical and numerical solutions to nonlinear problems arising in applied mathematics, Newtonian and non-Newtonian fluids, heat transfer analysis, magnetohydrodynamics, porous medium, mass transfer, and stagnation in stretching/shrinking sheet. He is also an author and co-author of more than 100 SCI-Publications. 